\title{
The Structure and Development of an Extratropical Cyclone over Northeastern Asia
}

\author{
Ying Liu ${ }^{1}$, Donghai Wang ${ }^{1,2}$, Zhaoming Liang ${ }^{1}$, and Chongjian Liu ${ }^{1}$ \\ ${ }^{1}$ State Key Laboratory of Severe Weather, Chinese Academy of Meteorological Sciences, Beijing, China \\ ${ }^{2}$ Guangdong Province Key Laboratory for Climate Change and Natural Disaster Studies, \\ School of Atmospheric Sciences, Sun Yat-sen University, Guangzhou, China
}

\begin{abstract}
The rare snowfall event in 2012 over North China due to the development of an unusual cyclone was analyzed. The results show that the cyclonic vorticity occurs first at the middle troposphere, and then extends to both the upper and lower troposphere. The trigger mechanism for the cyclone genesis is the baroclinic forcing with the upper unusual vorticity advection promoting its rapid development. The efficient deployment of the high- and lowlevel jets forms a favorable environment for its development and also transports a great number of vapor and the instability energy into the area of blizzard, resulting in the occurrence of the rare event.
\end{abstract}

(Citation: Liu, Y., D. Wang, Z. Liang, and C. Liu, 2016: The structure and development of an extratropical cyclone over northeastern Asia. SOLA, 12, 253-258, doi:10.2151/sola.2016-050)

\section{Introduction}

The wintertime severe snowfall in the most regions of the Northern Semisphere are generally associated with the extratropical cyclones' genesis and development (e.g. the President Day snowstorm of February 1979 in the Colombia region (Boast et al. 1984; Uccellini et al. 1985)). The recent relevant researches put the emphases on the formation process and structures differing from the classical ones (e.g., Martin 1998a and 1998b; Grim et al. 2007). In China, some of extraordinarily severe blizzard are also related with cyclone's formation and development. As an example, the strongest blizzard in the same period in history, in the eastern and southern Northeast China, was just caused by the Yangtz-Huanhe cyclone occurred on 3-5 March 2007 when moving northwards. Cai et al. (2009) and Liu et al. (2009) analyzed the synoptic background leading the cyclone to deepening and thus to the formation of the blizzard and, pointed out that the main causes that resulted in the explosive development of the cyclone were the distinct baroclinicity of the atmosphere and the strong temperature advection. On the other hand, Zhu et al. (2012) suggested that, based on the diagnoses using the generalized Z-O equation and the kinetic energy equation, the latent heating release and warm advection played a major role in the development of the cyclone at its initial stage while the latent heat releasing alone was the main forcing at the stage near its explosive deepening. Our study is concerned with a well-known case of the first mixed rainfall-snowfall event occurred in the late autumn, from 3 to 5 November 2012, due to the impact of the strong cold air burst and the development of an extratropical cyclone over North China (Wang et al. 2013). This study particularly analyzes the unusual structure of the associated cyclone and the complicated mechanism for its strengthening.

The analyses below are done based on the NCAR/NCEP reanalysis data (horizontal resolution $1^{\circ} \times 1^{\circ}$ ) and the conventional observation data in China. The relevant surface weather charts are from the Korea Meteorological Administration.

Corresponding author: Ying Liu, Chinese Academy of Meteorological Sciences, 46 Zhongguanchun South Ave., 100081 Beijing, China. E-mail: y119@camscma.cn. (22016, the Meteorological Society of Japan.

\section{Description of the case}

The first mixed rainfall-snowfall event in North China occurred during 3-5 November 2012, which is relatively earlier compared with the first snow events in other years. Figure 1 depicts the total precipitation from 0000 UTC 3 to 1200 UTC 4 November. The rainfall areas were widely distributed and the severe precipitation areas with a peak over $50 \mathrm{~mm}$ were mostly located from North China to the eastern coastal regions of the Yellow River and Huai River basins. The precipitation duration concentrated on the period from 0000 UTC 3 to 0000 UTC 4 during which the averaged precipitation amount over the Beijing area is $53.1 \mathrm{~mm}$ with the maximum of $96.0 \mathrm{~mm}$ occurred in the Haidian District.

The spatial distribution of the precipitation belts for this event was closely related to the cold air burst that was associated with the development of a cyclone with cold fronts was generated within a warm reverse-trough at surface (Fig. 2). The rainfall started from 0300 UTC 3 November and ended at 0600 UTC 4 November. Most of continuous precipitation occurred during the period of 0800 UTC 3-2000 UTC 3 with the maximum of $1 \mathrm{~h}$ accumulative precipitation appeared at 1000 UTC 3 . The temperature at the Beijing station began to go down rapidly from 0800 UTC 3 November and rebounded at 2100 UTC 3, with the minimum of $1^{\circ} \mathrm{C}$ occurred at 2000 UTC 3 . The temporal variations of pressure were similar to that of temperature with the lowest pressure emerged at 2100 UTC 3.

\section{Structures of the cyclone}

The distributions of temperature and geopotential height anomalies through the centres of the cyclone at the various constant-pressure layers for its different stages of evolution were calculated to reveal the thermodynamic structures. The compu-

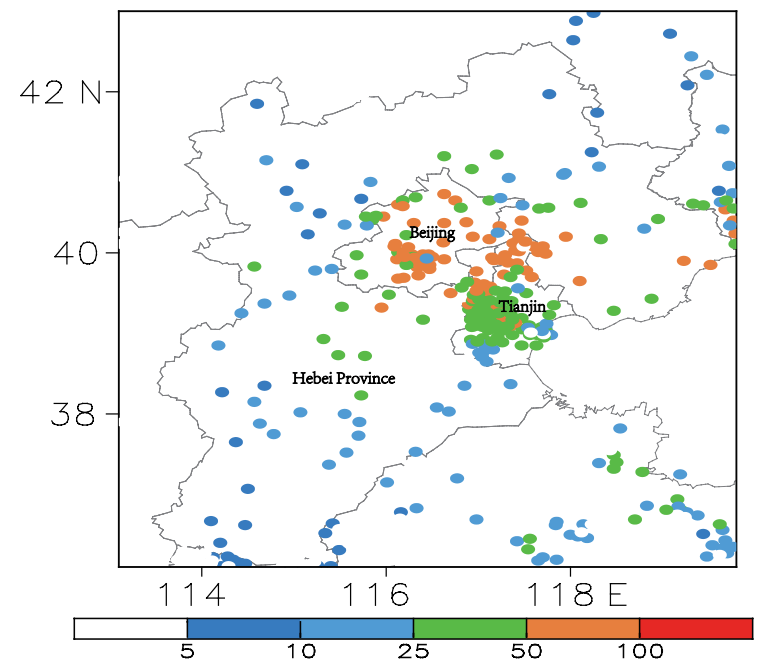

Fig. 1. Accumulative precipitation (mm) from 0000 UTC 3 to 1200 UTC 4 November 2012 around the Beijing-Tianjin-Hebei Province area. 

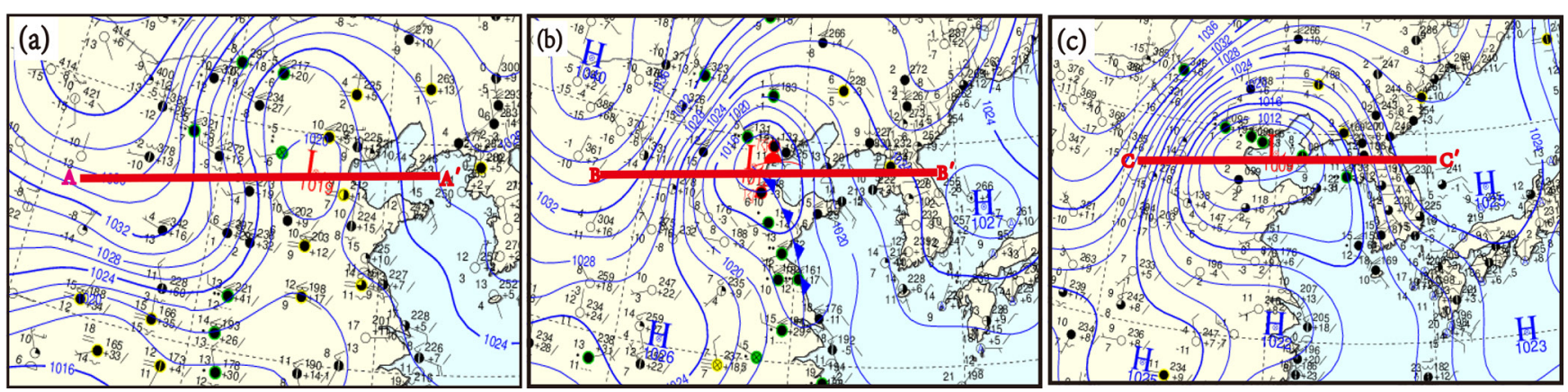

Fig. 2. Surface weather charts (courtesy of the Korea Meteorological Administration) at (a) 0000 UTC 03, (b) 1200 UTC 03 and (c) 0000 UTC 04 November with the red dashed line in each panel being for the position through which the cross sections of Fig. 4 are made respectively.

tational procedure for the anomalies are as follows: calculating first the averaged geopotential height/temperature values over the respective constant-pressure layer within the domain under study, and subtracting then the averaged values from the corresponding geopotential height/temperature values at the points of the respective constant-pressure layer to get the geopotential height/ temperature anomalies fields. It is found that, as usually, in the lower layer $(850 \mathrm{hPa}$, figures not presented), at the initial stage of the cyclone evolution, the cold centre is located behind the geopotential height centre which is closer to its corresponding centre of pressure at the surface. For the middle troposphere $(500 \mathrm{hPa}$, figures not presented) the situation is different where the cold centre tends to coincide with that of geopotential height with the lapse of time. The prominent difference from a usual extratropical cyclone in terms of the temperature/pressure structures lies in its large vertical range as shown in Fig. 3 .

It is distinctly seen from Fig. 3 that the cyclone extends to a higher level in the stratosphere, which is quite different from the general extratropical cyclone and very similar to the counterpart in higher latitudes, say, the Northeast low, which is very deep in terms of the vertical thickness. This interesting characteristic might be associated with the distribution of vertical motion (figures not presented), an apparent factor causing the severe snowfall.
Figure 4 shows the cross sections of the vertical velocity. It is seen from Fig. 4 that the vertical velocities in the middle and lower troposphere have been gradually organized with the lapse of time. At the initial stage (Figs. $4 \mathrm{a}$ and $\mathrm{b}$ ) the maximum updraft of $-1 \mathrm{hPa} \mathrm{s}^{-1}$ occurred between 500 and $700 \mathrm{hPa}$, which is located behind the cold front, with a little disorder to a certain extent. However, the air current is well organized at the strengthening stage (Figs. 4c and d), which is associated with a back-slanted vertical motion from the surface to $300 \mathrm{hPa}$ with its maximum velocity reaching $-2 \mathrm{hPa} \mathrm{s}^{-1}$. At the same time, the up-branch of the secondary circulation becomes narrow and stronger with the vapor lifted along the front and climbed slantly. This branch bifurcates when reaching the middle levels with one of them outflowing anticyclonically as it is brought to the higher levels where the relative humidity pattern shows a form of anvil, and, another branch brought to the ground in a way of cyclonic rotation in its rear where it meets the subsidencing cold air. In the meantime, the precipitation becomes heavier with its width narrowed. Finally at the prime stage (Figs. 4e and f), ascending motions occur largely in the lower troposphere with the intensity weakened when the cold air has invaded the area near the cyclone's centre with the precipitation tending to become decreased.
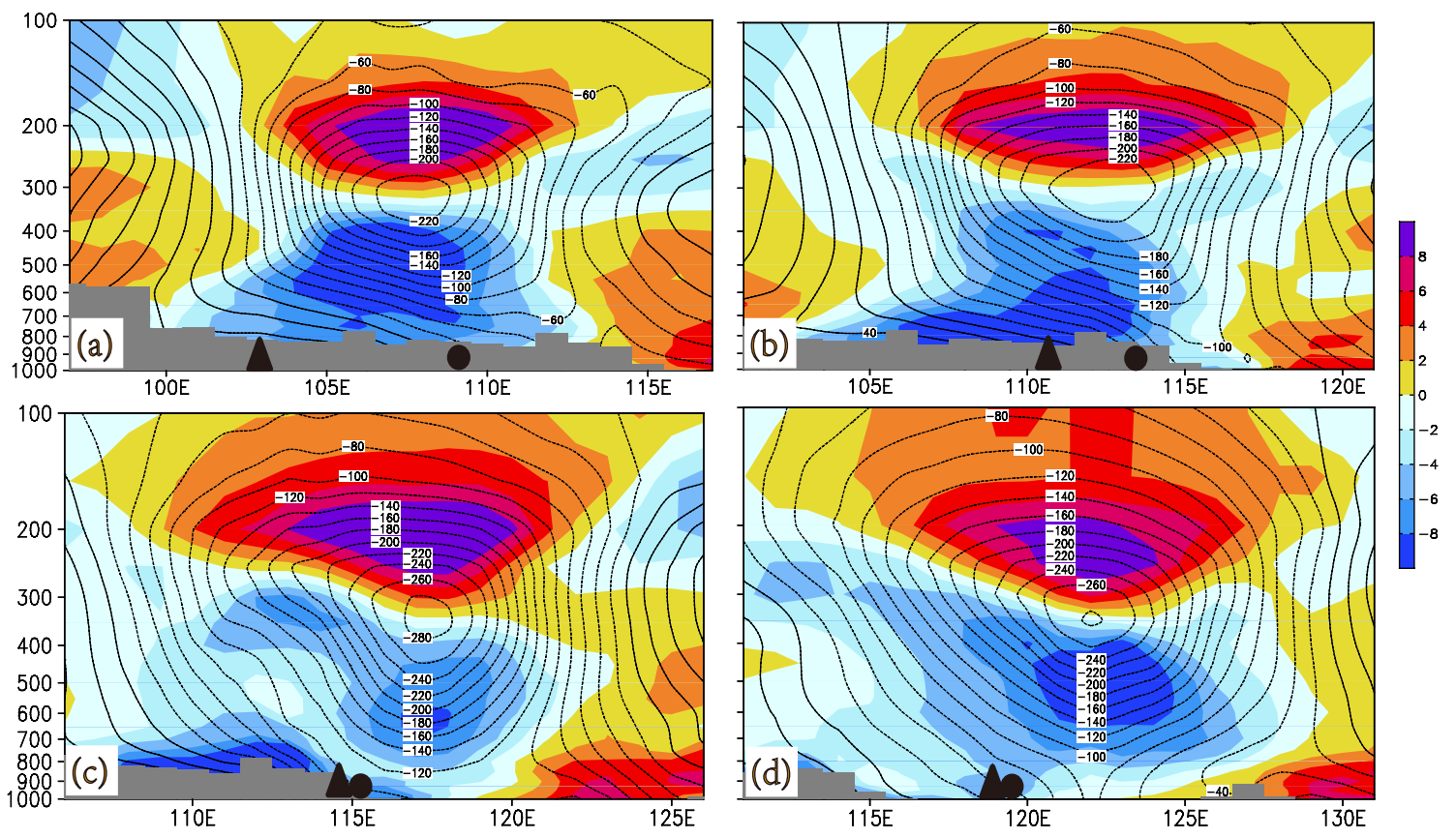

Fig. 3. Vertical cross sections of temperature anomalies (shaded, unit: ${ }^{\circ} \mathrm{C}$ ) and geopotential height anomalies through the cyclone's centre for the different stages ( contour, unit: gpm; and $\boldsymbol{\Delta}$ represent the central position of the cyclone at $500 \mathrm{hPa}$ and surface, respectively). (a) 0000 UTC 3, (b) 1200 UTC 3, (c) 0000 UTC 4, and (d) 0000 UTC 5 November. 

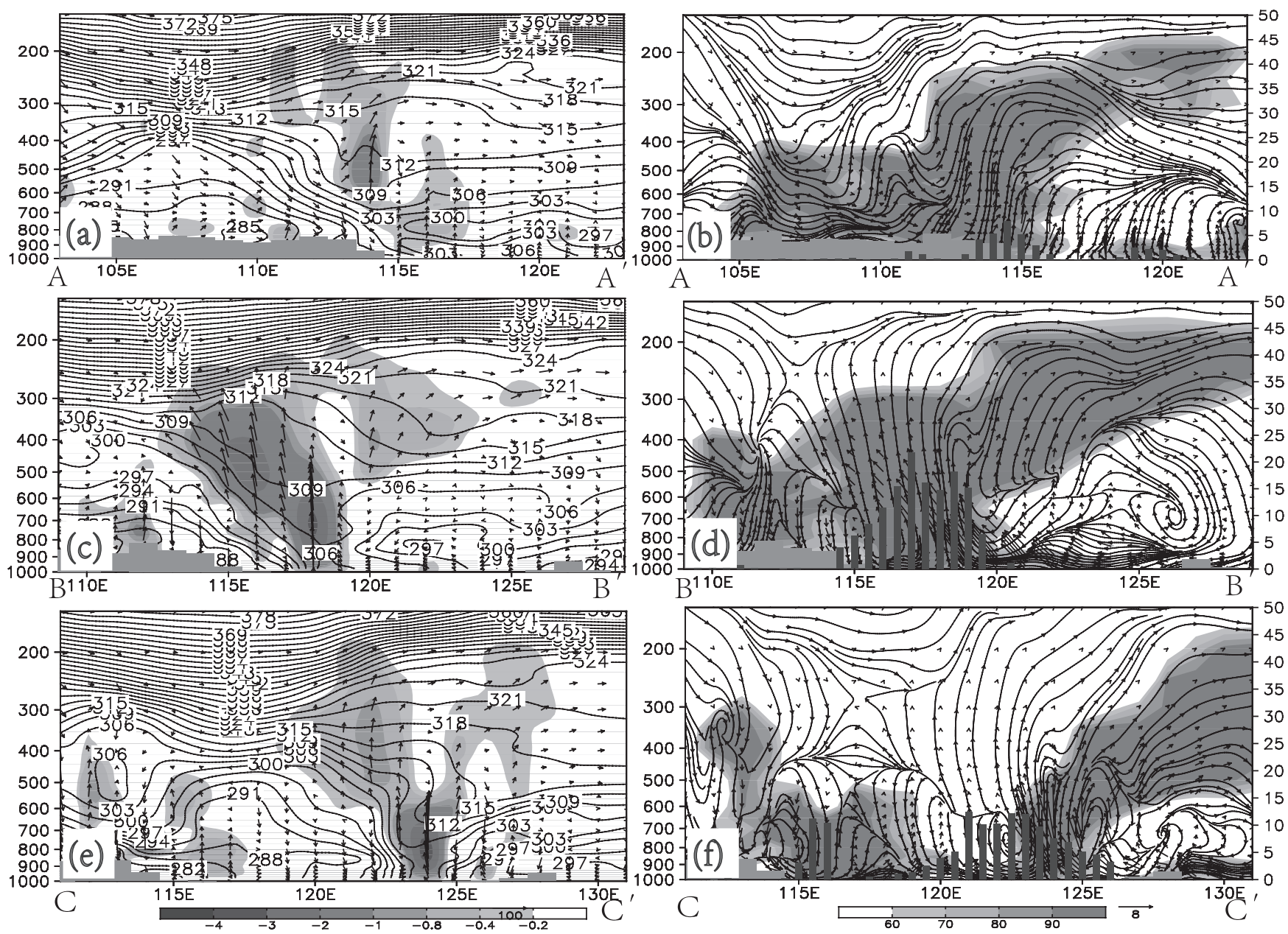

Fig. 4. (a, c, e) Vertical cross sections of vertical velocity (shaded, hPa s ${ }^{-1}$ ), pseudo-equivalent potential temperature (contour, K) through the center of the cyclone for its different stages; (b, d, f) as in (a), but for relative humidity (shaded, \%), vertical circulation (streamline) and water vapour flux (vector, $\mathrm{g} /(\mathrm{cm} \cdot \mathrm{hPa} \cdot \mathrm{s})$ and the corresponding thenceforth $6 \mathrm{~h}$ accumulative rainfall (histogram, mm). (a)/(b) 0000 UTC 3 , (c)/(d) $1200 \mathrm{UTC} 3$, and (e)/(f) 0000 UTC 4 November. The cross sections are made through the red dashed line in the corresponding panel in Fig. 2 respectively.

\section{Development mechanism of the cyclone}

\subsection{Effects of vorticity advection and temperature advection}

The analysis of the evolution of the cyclone shows that the development of the cold vortex in the higher troposphere is related to the cyclone's strengthening at the surface with the cold vortex's development promoting the intensity of the surface's cyclone. It can be seen from the every $6 \mathrm{~h}$ evolution of the vorticity advection and temperature advection at $300 \mathrm{hPa}$ (figures not presented) that the positive vorticity advection at $300 \mathrm{hPa}$ reaches its maximum at 0600 UTC 3 and the corresponding temperature advection is at its prime at 0000 UTC 4 with the surface's cyclone deepening rapidly after 1200 UTC 3 and reaching its prime at 0000 UTC 4. Consequently, the cyclone at its mature stage shows a structure of cold core in the middle-lower troposphere and warm core in the upper troposphere. As a result, the warm advection enhanced gradually in the upper troposphere might be a major factor causing the temperature centre at a higher level to form.

Compared with the upper troposphere, the temperature at $850 \mathrm{hPa}$ is much lower (Fig. 5), suggesting that the effect of temperature advection in the lower troposphere is relatively quite weak. However, as the cyclone develops, it causes the cold air behind the cyclone to flow into its centre and the warm-humid currents in the front of it to converge. This leads the convergence in the lower troposphere to strengthening and thus benefiting the development of the cyclone. In the meantime, the warm advection and the cold advection rotate mutually with the results that the areas of warm/cold advection are interrupted apart from the warm/ cold currents, causing eventually the cyclone to stop developing owing to the decreasing of warm/cold advections.

The analyses above show that the evolutionary characteristics of this cyclone is very special, which are different from neither the class A of cyclone nor the class B, as summarized by Petterssen (1971).

\subsection{Analysis of the diabatic heating}

Some previous researches pointed out that latent heat release plays an important role in the occasion when a cyclone enters seas at the moment around its explosive development period (Zhao et al. 1994). On the other hand, the potential vorticity is a physical variable that combines the thermal effect with the dynamic one, and able to more completely describe the genesis and development of a cyclone as well as the associated severe precipitation. The extratropical cyclone under study is associated with severe snowfall as is rarely met in late autumn in northern China, which should cover some particular thermal and dynamic processes in terms of potential vorticity. Below we will investigate the evolutionary characteristics of the wet potential vorticity based on the calculations of the relevant diagnostic formulation, which can be found in (e. g.) Liu et al.(2012).

The corresponding diagnostic formulas for the apparent heat source $\left(Q_{1}\right)$ and apparent moisture sink $\left(Q_{2}\right)$ in the p-coordinates can be written as 

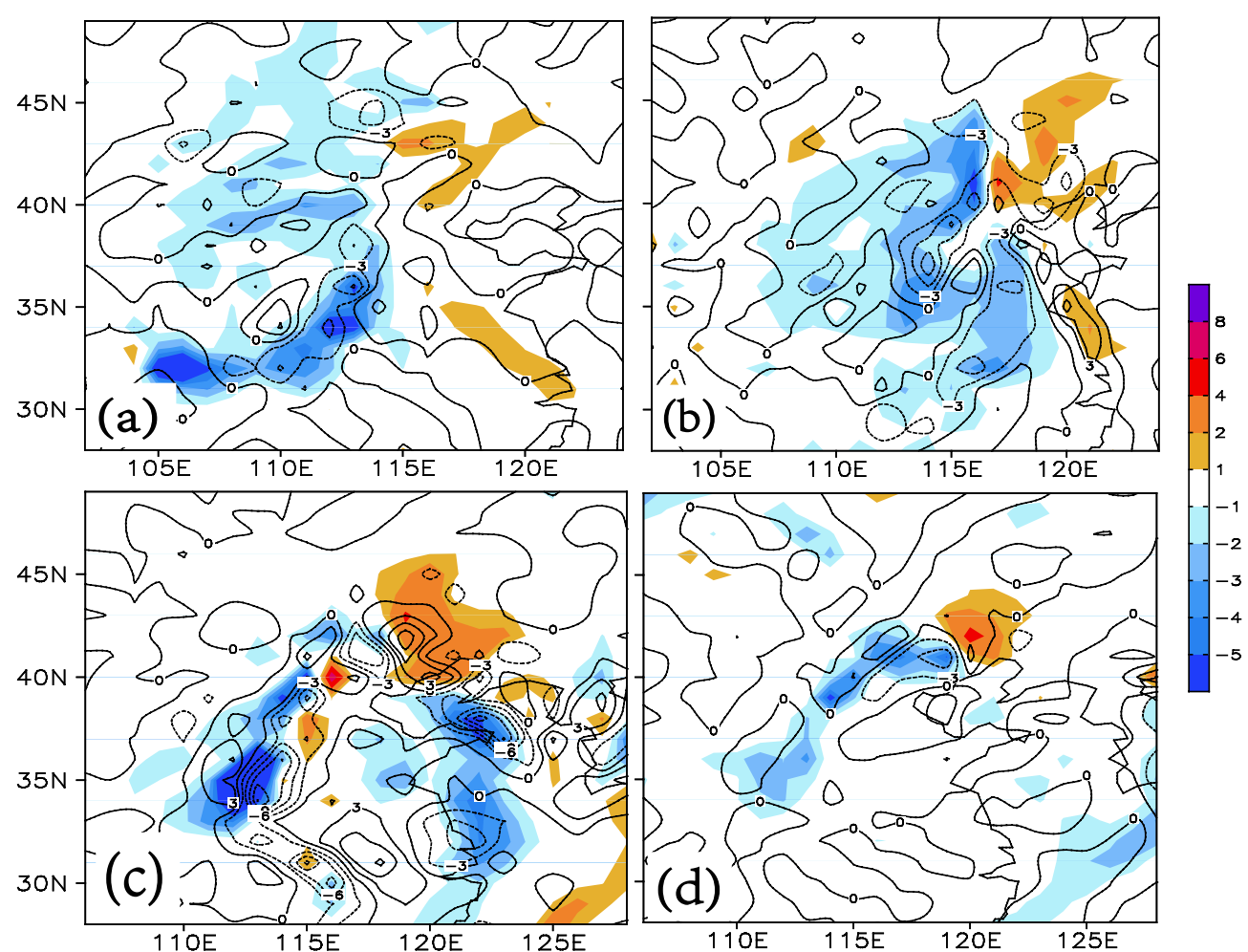

Fig. 5. Distributions of temperature advection (shaded, $10^{-4} \mathrm{~K} \mathrm{~s}^{-1}$ ) and vorticity advection (contour, $10^{-9} \mathrm{~s}^{-2}$ ) at $850 \mathrm{hPa}$ for (a) 0000 UTC 3, (b) 1200 UTC 3, (c) 0000 UTC 4, and (d) 0000 UTC 5 November.

$$
\begin{aligned}
& Q_{1}=C p\left[\frac{\partial T}{\partial t}+\vec{V} \cdot \nabla T+\left(\frac{p}{p_{0}}\right)^{\kappa} \omega \frac{\partial \theta}{\partial p}\right] \\
& Q_{2}=-L\left[\frac{\partial q}{\partial t}+\vec{V} \cdot \nabla q+\omega \frac{\partial q}{\partial p}\right]
\end{aligned}
$$

where $L$ is the latent coefficient, $\theta$ potential temperature, $q$ specific humidity, $v$ horizontal velocity, $\kappa=\mathrm{R} / c_{p}, R$ and $c_{p}$ are respectively gas constant and specific heat at constant pleasure, and $\omega$ vertical velocity. $Q_{1}$ and $Q_{2}$ are the warming rate for air per unit mass and the rate caused by vapor per mass owing to condensation, respectively, which both are in $\mathrm{J} /(\mathrm{kg} \cdot \mathrm{s})$. For the sake of being directly perceived through the senses, they are displaced by $Q_{1} / c_{p}$ and $Q_{2} / c_{p}$ for describing the evolutionary characteristics of the so-called apparent heat source and the apparent moisture sink during the cyclone's life-cycle, respectively, which are in $K d^{-1}$. In the following text, for simplicity, we simply name $Q_{1}$ and $Q_{2}$ as the apparent heat source and the apparent moisture sink, respectively.

To further illustrate the relationship of diabatic heating with the cyclone evolution, a number of profiles are made through an averaging $Q_{1}$ and $Q_{2}$ calculation over a rectangle consisting of an area covering respectively 5 longitudes in the west and the east as well as 3 latitudes in the north and the south. Figure 6 shows the vertical profiles for the area-averaged $Q_{1}, Q_{2}$ and their components in which $Q_{11}, Q_{12}$, and $Q_{13}$ stand for the terms of local change, horizontal advection and vertical transfer in Equation (1), respectively, and, similarly, $Q_{21}, Q_{22}$, and $Q_{23}$ for those respectively in Equation (2). As a whole, at the initial stage (Fig. 6a), latent heat contribution is small and sensible heat one concentrates on the lower troposphere (notice that below $400 \mathrm{hPa}$ it is positive) with the peak at $600 \mathrm{hPa}$. At the stage of rapid development (Fig. 6b) there exist two peaks in $Q_{2}$ which are located respectively near the ground and at $600 \mathrm{hPa}$ at which condensation emerges. The relative cold layer occurs at $750 \mathrm{hPa}$ and thus a potential instability layer forms in the mid-lower levels owing to warming zone situated near the ground, which is just the unstable stratification as a cause for the severe snowfall to occur. The profiles for $Q_{1}$ are very similar to $Q_{2}$ with the peak at $600 \mathrm{hPa}$ being distinctly larger than $Q_{2}$, implying that condensation is a major contribution in the lower troposphere. Compared with the earlier stages, the profiles of $Q_{1}$ and $Q_{2}$ at the mature stage (Fig. 6c) vary dramatically especially with the contribution of $Q_{2}$ near the ground being negative. Among it the horizontal advection term shows a negative contribution even for all the layers and the horizontal advection component of $Q_{1}$ below $750 \mathrm{hPa}$ becomes a weak negative contribution, meaning that the lower troposphere has been governed by cold advection. And, at the decay stage (Fig. 6d), the resultant contribution of both $Q_{1}$ and $Q_{2}$ is negative, implying that the mid-lower layers of the cyclone are full with cold air and it has come to its decay stage.

Most significantly, the above analysis shows that the vapor condensation has an important impact on the development of the cyclone. In particular, at the stage of rapid development, the strong advection effect of the warm-wet currents causes the warming and humidifying in the lower levels and thus constructs an unstable layer against the middle layer of relative coldness, which is a favorable condition for generating severe snowfall.

\subsection{Relationship of upper-level jets with the development of the cyclone}

Figure 7 shows the comprehensive dynamic fields where the middle-level $(500 \mathrm{hPa})$ geopotential height superposed by both the upper-level $(300 \mathrm{hPa})$ and lower-level winds $(925 \mathrm{hPa})$. It is seen from Fig. 7 that there exist two branches of upper-level jets with the northern branch of polar front jet convex to the south owing to upper-level low vortex's existence whose northern end is situated at $38^{\circ} \mathrm{N}$ and, the southern branch of subtropical westerlies jet parallel nearly to latitudes (Fig. 7a). The surface cyclone is located at the right side of the northern branch with the distance of about 5 longitudes between the cyclone and the jet. On the other hand, at the very low levels (say, $925 \mathrm{hPa}$ ) there also is a jet with its orientation of southeast by south that spreads from the East China 

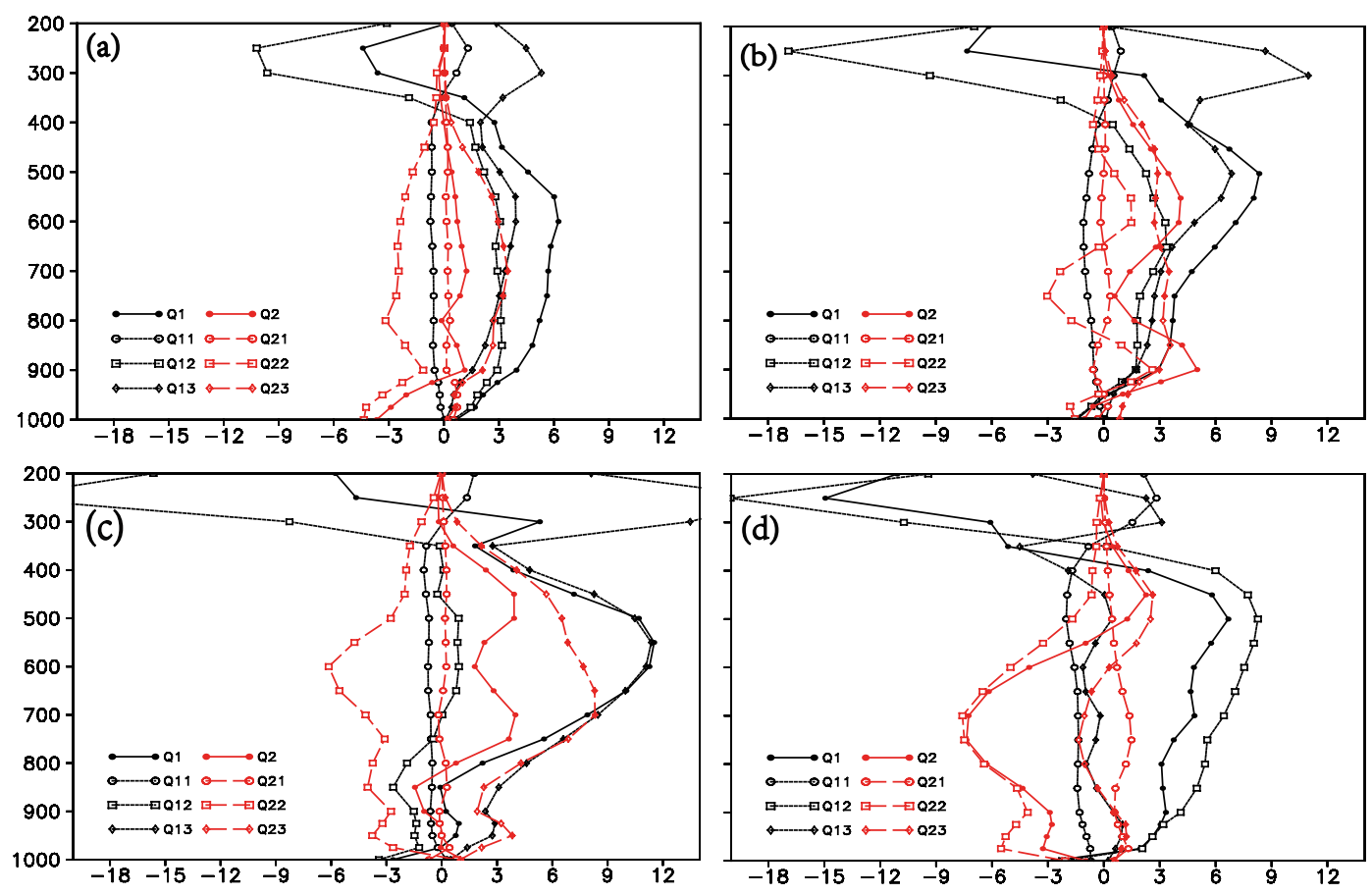

Fig. 6. Vertical profiles for the area-averaged $Q_{1}, Q_{2}$ and their components $(\mathrm{K} / \mathrm{d})$

(a) 0000 UTC 3, (b) 1200 UTC 3, (c) 0000 UTC 4, and (d) 0000 UTC 5 November
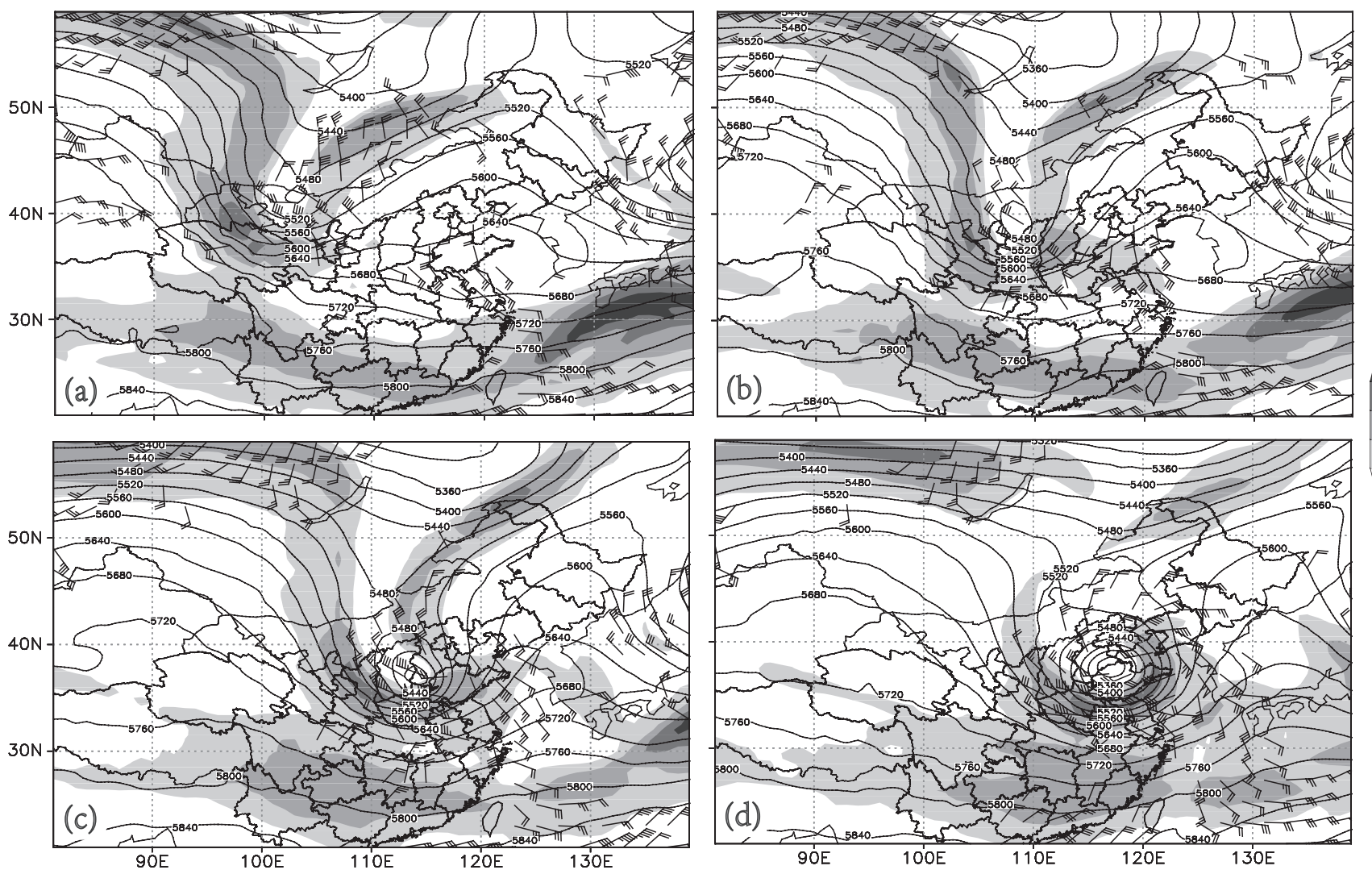

Fig. 7. Geopotential height at $500 \mathrm{hPa}$ (contour, gpm) superposed by the horizontal winds at $300 \mathrm{hPa}$ (shaded $\geq 30 \mathrm{~m} \mathrm{~s}^{-1}$ ) and at $925 \mathrm{hPa}\left(\right.$ barb $\geq 12 \mathrm{~m} \mathrm{~s}{ }^{-1}$ ) for (a) 1200 UTC 2, (b) 0000 UTC 3, (c) 1200 UTC 3, and (d) 0000 UTC 4 November. 
Sea to Shandong Peninsula with its front edge reaching at the northern part of Shandong Peninsula at 1200 UTC 2. The upperlevel polar front jet has made a rapid shift eastward till 0000 UTC 3 and, in the meantime, the front edge of the lower-level jet moves further northward into the southern part of northern China with the surface cyclone situated at the right side of the upper-level jet as well as the leading end of the lower-level jet (Fig. 7b). During this period, the cold front moves eastward to the neighbourhoods of eastern Shanxi Province - Henan Province - western Hubei Province when such an upper- and lower-level collocation is favorable to enhancing the lower-level convergence and thus stimulating the development of the cyclone on the lower levels. At 1200 UTC the cold front shifts to the surroundings of Shandong Peninsula - Jiangsu Province - the Fujian coast areas, causing the northerly area behind the front to shift eastward, and thus leading to its confluence with the southerly jet over northern China. This benefits the further strengthening of the lower convergence. Meantime there still exists the upper-level divergence due to the surface low's centre situated also at the right side of the northern branch of jet (Fig. 7c), which results in the rapid development of the cyclone because of the combining effect of the upper-level divergence with the intensifying lower-level convergence. At 0000 UTC the northern branch of polar front breaks (Fig. 7d) whose northern end joins with the southern branch of the jet. At that time the surface cyclone's centre is situated at the left side of the upper-level axis of jet so that the upper-level effect of divergence weakens, leading the cyclone's development to ceasing.

The above analysis shows that the development of the cyclone is significantly related with the development and evolution of the upper- and lower-level jets as well as their deployment against each other. On the other hand, not only the southerly currents in the lower levels transport moisture and heat to the area of snowfall but also their confluence with cold air brought by the northerly behind the front causes strong ascending motion, resulting thus in severe precipitation processes. It is then suggested that the increase of vorticity associated with the convergence at the front end of the low level jet serves as an important factor responsible for the development of the cyclone.

\section{Conclusive remarks}

The analyses above reveal that this case of extratropical cyclone shows unusual thermal and dynamic structures as well as the complicated mechanism for the rare severe snowfalls/rainfalls emergence in the late autumn in northern China. The cyclone generates within a warm reverse-trough at surface and develops into a mature cold vortex system extended vertically to a very higher level. It, as a deep cold low system, is similar to Northeast cold vortex in terms of thermodynamic structure. During its development process, the cold centre in the higher levels shifts gradually to the lower levels, suggesting that the cold airs at upper levels sink toward the ground, which plays an important role in the cyclone's deepening. On the other hand, the vorticity advection at the upper levels reaches its prime just before the rapid development of the cyclone, implying that the upper level vorticity advection is a major cause for the cyclone's rapid development. The other key factors cover the appropriate lower level warmwet advection and advantageous deployment of the low level jet against the upper level one. The former promotes an unstable stratification to form as it is superposed by a middle level layer of cold air, and the later constructs favourable conditions of divergence in the upper levels and convergence in the lower levels. It might be one of the most significant mechanisms for the fact that the obvious increase of vorticity caused by the convergence at the front end of the southerly jet at the very low level transfers a large number of moisture and unstable energy to the area of severe snowfall, resulting in the rare severe snowfall to emerge.

\section{Acknowledgements}

This work was jointly supported by the National Natural Science Foundation of China under Grant Nos. 41075048 and 91437221, the National Key Basic Research Program of China under Grant No. 2012CB417204, the R\&D Special Fund for Public Welfare Industry (meteorology) by the Ministry of Finance and the Ministry of Science and Technology under Grant Nos. GYHY201006006, GYHY201306004 and GYHY201506002.

Edited by: K. Kawamoto

\section{References}

Bosart, R. J., and S. C. Lin, 1984: Diagnostic analysis of the president day snowstorm of February 1979. Mon. Wea. Rev., 112, 2148-2177.

Cai, L. N., Y. J. Sui, D. Q. Liu, S. Wang, W. Liu, and T. K. Wang, 2009: Analysis on an unusual snowstorm event caused by explosive cyclone. Acta Scientiarum Naturalium Universitatis Pekinensis, 45, 693-700.

Grim, J. A., R. M. Rauber, M. R. Ramamurthy, B. F. Jewelt, and M. Han, 2007: High-resolution observations of the trowalwarm-frontal region of two continental winter cyclones. Mon. Wea. Rev., 135, 1629-1646.

Liu, N. W., L. L. Qi, and J. W. Han, 2009: The analyses of an unusual snowstorm caused by the northbound vortex over Liaoning Province in China. Chinese J. Atmos. Sci., 32, 274284.

Liu, Y., D. H. Wang, Z. F. Zhang, and S. X. Zhong, 2012: A comprehensive analysis of the structure of a northeast Chinacold-vortex and its characteristics of evolution. Acta Meteor. Sin., 70, 354-370.

Martin, J. E., 1998a: The structure and evolution of a continental winter cyclone. Part I: Frontal structure and the occlusion process. Mon. Wea. Rev., 126, 303-328.

Martin, J. E., 1998b: The structure and evolution of a continental winter cyclone. Part II: Frontal forcing of an extreme snow event. Mon. Wea. Rev., 126, 329-348.

Petterssen, S., and S. J. Smebye, 1971: On development of extratropical cyclones. Quart. J. Roy. Meteor. Soc., 97, 457-482.

Uccellini, L. W., K. F. Keyser, K. F. Brill, and C. H. Wash, 1985: The president day cycle of 18-19 February 1979: Influence of upstream trough implication and associated tropopause folding on rapid cyclogenesis. Mon. Wea. Rev., 113, 962988.

Wang, D. H., Y. H. Duan, Y. Liu, Z. M. Liang, C. J. Liu, Y. F. Zhao, Y. W. Zhang, J. F. Yin, and H. Wang, 2013: A case study of the mixed rainfall-snowfall event associated with an extratropical cyclone in autumn. Acta Meteor. Sin., 71, 606627.

Zhao, Q. G., Q. J. Yi, and Y. H. Ding, 1994: A dynamic study of an explosive development of marine extratropical cyclone. Acta Oceanol. Sin., 16, 30-37.

Zhu, Y. L., S. L. Zhou, Q. F. Lin, and Z. M. Wu, 2012: Dynamic and thermal analyses on an rapidly developing extratropical cyclone after entering Sea. Plateau Meteor., 31, 788-797.

Manuscript received 11 April 2016, accepted 12 August 2016

SOLA: https://www.jstage.jst.go.jp/browse/solal 